\title{
RDAU-Net: Based on a Residual Convolutional Neural Network With DFP and CBAM for Brain Tumor Segmentation
}

\author{
Jingjing Wang ${ }^{1}$, Zishu $Y u^{1}$, Zhenye Luan ${ }^{1}$, Jinwen Ren ${ }^{1}$, Yanhua Zhao ${ }^{2 *}$ and Gang $Y u^{1 *}$ \\ ${ }^{1}$ College of Physics and Electronics Science, Shandong Normal University, Jinan, China, ${ }^{2}$ Obstetrics and Gynecology, \\ Tengzhou Xigang Central Health Center, Tengzhou, China
}

\section{OPEN ACCESS}

Edited by:

Kyung Hyun Sung,

UCLA Health System,

United States

Reviewed by:

Jue Jiang,

Memorial Sloan Ketter Cancer

Center, United States

Weiwei Zong,

Henry Ford Health System,

United States

*Correspondence:

Yanhua Zhao

13561119458@163.com

Gang Yu

yugang2011@foxmail.com

Specialty section:

This article was submitted to

Cancer Imaging and

Image-directed Interventions,

a section of the journal

Frontiers in Oncology

Received: 30 October 2021 Accepted: 14 January 2022

Published: 02 March 2022

Citation:

Wang J, Yu Z, Luan Z, Ren J, Zhao Y and Yu G (2022) RDAU-Net: Based on

a Residual Convolutional Neural

Network With DFP and CBAM for

Brain Tumor Segmentation.

Front. Oncol. 12:805263.

doi: 10.3389/fonc.2022.805263
Due to the high heterogeneity of brain tumors, automatic segmentation of brain tumors remains a challenging task. In this paper, we propose RDAU-Net by adding dilated feature pyramid blocks with 3D CBAM blocks and inserting 3D CBAM blocks after skipconnection layers. Moreover, a CBAM with channel attention and spatial attention facilitates the combination of more expressive feature information, thereby leading to more efficient extraction of contextual information from images of various scales. The performance was evaluated on the Multimodal Brain Tumor Segmentation (BraTS) challenge data. Experimental results show that RDAU-Net achieves state-of-the-art performance. The Dice coefficient for WT on the BraTS 2019 dataset exceeded the baseline value by $9.2 \%$.

Keywords: brain tumor segmentation, U-Net, attention mechanism, dilation feature pyramid, deep learning

\section{INTRODUCTION}

Tumors that grow in the skull are commonly referred to as brain tumors and include primary brain tumors, which occur in the brain parenchyma, and secondary brain tumors, which metastasize to the skull from other parts of the body. According to the World Health Organization (WHO) classification criteria, brain tumors are classified into four grades: grade I, astrocytoma; grade II, oligodendroma gliomas; grade III, anaplastic glioma; and grade IV, glioblastoma multiforme (GBM) (1). The lower the grade of the tumor, the less malignant it is, and the better the prognosis is. As a result, early diagnosis of brain tumors is very important for treatment.

Magnetic resonance imaging (MRI) is considered a standard technique due to its satisfactory soft-tissue contrast and wide availability (2). MRI is a noninvasive imaging technique that uses magnetic resonance phenomena to obtain electromagnetic signals from the human body and reconstruct information about the body as a type of tomography. MRI is available in a variety of imaging sequences. These imaging sequences can produce MRI images with distinctive features that can reflect the anatomical morphology of the human body.

In current clinical practice, brain tumors are labeled manually by physicians, which is timeconsuming. Moreover, brain tumors are similar to normal brain tissues in terms of morphology and 
intensity; hence, manual labeling by physicians suffers from subjective variability and lacks reproducibility (3). Therefore, accurate automatic segmentation of brain tumors in T1, T1-c, T2, and FLAIR is essential for quantitative analysis and evaluation of brain tumors (4).

\section{Progress on Image Segmentation Methods}

In recent years, deep neural network (DNN)-based methods have achieved high performance for brain tumor segmentation (5-8). Convolutional neural networks (CNNs) (9) have achieved great success in many research areas, such as image recognition (10-12), image segmentation (13-15), and natural language processing $(16,17)$. In (18), a fully convolutional neural network (FCN) for image pixel-level image classification was proposed, which solves the problem of semantic-level image segmentation with input data of arbitrary size. Ronneberger et al. (19) proposed the U-Net framework with a skip connection module connecting the encoder and decoder. In contrast to FCNs, U-Net fuses shallow and deep features and has produced impressive results in medical image segmentation. Inspired by U-Net, Attention U-Net (20) and ResU-Net (21) were proposed and used for medical image segmentation. In Attention U-Net, an attention mechanism is added to the skip connection part. This module generates gating information to readjust the weight coefficients of features at various spatial locations. In ResU-Net, each convolutional layer is replaced with a residual convolutional layer, thereby avoiding gradient disappearance in backpropagation in deep network structures.

\section{Attention Mechanism}

Attention mechanisms were first introduced in natural language processing (22-25). Currently, attention mechanisms are also widely used in deep learning to enhance feature extraction (2628). Hu et al. (29) proposed plug-and-play squeeze-and-excitation (SE) attention, which learns feature relationships to obtain contextual information on channel dimensions by global average pooling. Wang et al. (30) proposed ECANet, which uses a local cross-channel interaction strategy without downscaling and adaptive selection of one-dimensional convolutional kernels. In addition to these single-channel attention mechanisms, there are several dual-attention mechanisms. For example, Fu et al. (31) proposed the dual attention mechanism network (DANet) to improve the accuracy of network segmentation by capturing feature dependencies based on the spatial and channel dimensions of the self-attention mechanism and summing the outputs of the two modules. Woo et al. (32) proposed a convolutional block attention module (CBAM) to enhance useful information and suppress useless information by a tandem channel attention mechanism and a spatial attention mechanism.

Although ResU-Net uses a residual module to mitigate the problem of vanishing network gradients, it still suffers from the following problems: (1) Multiscale features have an important role, but ResU-Net does not extract features from images of various sizes, and thus, a substantial amount of detailed information is lost. (2) The skip connection cascades the shallow features of the decoder part and the corresponding depth features to achieve feature fusion, but the shallow features of the encoder contain considerable redundant information, which, in turn, affects the segmentation results.

In this paper, we propose RADU-Net, which is an improved version of ResU-Net that is inspired by the attention mechanism. Our contributions are mainly as follows.

1. We insert the 3D CBAM dual attention mechanism in each residual module to alleviate the problem of gradient disappearance or explosion as the network structure deepens and obtain the feature information of the image more accurately.

2. We add the dilated feature pyramid module with the $3 \mathrm{D}$ CBAM dual attention mechanism between the encoder and the decoder as a solution to the problem that the traditional U-Net network does not extract multi-scale features of images to obtain feature maps of different sizes.

3. We insert a 3D CBAM block after the skip connection in each layer to improve the extraction of channel information and spatial information to reduce the redundant information of low-level features.

Via these modifications, RADU-Net solves the above problems and improves the overall segmentation accuracy of brain tumors.

\section{METHODS}

\section{RDAU-Net}

This paper proposes a 3D convolutional neural network, namely, RDAU-Net, for the brain tumor segmentation task. The 3D CNN considers more comprehensive spatial context information and achieves more accurate performance than the $2 \mathrm{D} \mathrm{CNN}$ in image segmentation. Figure 1 illustrates the complete structure of RDAU-Net. RDAU-Net includes an encoder part and a decoder part. The input of the encoder part includes 2 purple $3 \mathrm{D}$ convolutional layers with a convolutional kernel size of $3 \times$ $3 \times 3,53 \mathrm{D}$ RA blocks of various sizes and $43 \mathrm{D}$ convolutional layers with a step size of 2 as the downsampling layer. The RA block is that the orange CBAM block is added after each convolutional layer of the residual block for feature extraction. Between the encoder and the decoder is the DA block, which is the CBAM block that is added after each dilated convolution layer. The decoder part is symmetric to the encoder and contains 4 deconvolution layers. The purple arrow between the encoder and decoder is the skip connection, and a CBAM block is inserted after each skip connection layer. The pink block is the concatenation layer. The network ends with a $3 \mathrm{D}$ convolutional layer and a gray block of sigmoid function layers. $(3,3,3,4,32)$ in the first layer indicates that the convolution kernel size of this layer is $3 \times 3 \times 3$, the number of input features is 4 and the number of output features is 32 .

\section{D CBAM Block}

To extract more accurate image feature information, we use CBAM (32) as the attention module of the network. We transform the 2D CBAM attention module into a 3D CBAM 


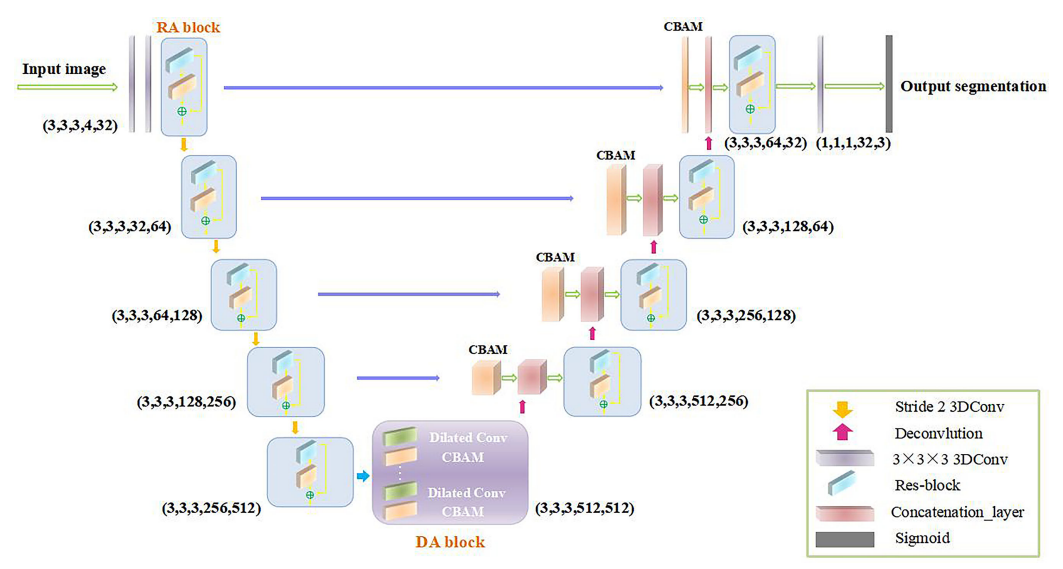

FIGURE 1 | Architecture of the proposed RDAU-Net.

attention module. CBAM ties together channel attention and spatial attention to increase the weights of useful features in the channel and useful features in the space, as illustrated in Figure 2A. First, the deep blue module in Figure 2A, which is denoted as $F \in R^{W \times H \times D \times C}$, is input into the channel attention module. The channel attention module as shown in Figure 2B consists of a $3 \mathrm{D}$ global max pooling module (the pink module in Figure 2B), a 3D global average pooling module (the yellow module in Figure 2B), and a shared MLP (multilayer perception) that consists of a $3 \mathrm{D}$ neural network. The input feature map $\mathrm{F}$ is subjected to $3 \mathrm{D}$ global max pooling and $3 \mathrm{D}$ global average pooling operations and to MLP. The two outputs of MLP are added elementwise. Then, after the sigmoid activation function, we obtain the weight $M_{c}(F)$ after the channel attention module.

$$
\begin{aligned}
M_{C}(F) & =\sigma(M L P(\text { AvgPool } 3 D(F)+M L P(\operatorname{MaxPool} 3 D(F)) \\
& =\sigma\left(W _ { 1 } \left(W_{0}\left(F_{\text {avg }}^{C}\right)+W_{1}\left(W_{0}\left(F_{\max }^{C}\right)\right)\right.\right.
\end{aligned}
$$

where $W_{0} \in R^{C / r \times C}, W_{1} \in R^{C / C \times r}$, and $\sigma$ is the sigmoid activation function. $r$ takes a value of 16 , namely, the channel $C$ is changed to $C / 16$ during max-pooling and average pooling to reduce the number of parameters.

Then, $M_{c}(F)$ is multiplied with the input feature map $F$ to obtain the output feature map $F^{\prime}$ of the channel attention module, and the formula is as follows:

$$
F^{\prime}=M_{C}(F) \otimes F
$$

where $M_{c}(F)$ denotes the output weight after the channel attention module and $\otimes$ denotes element-by-element multiplication.

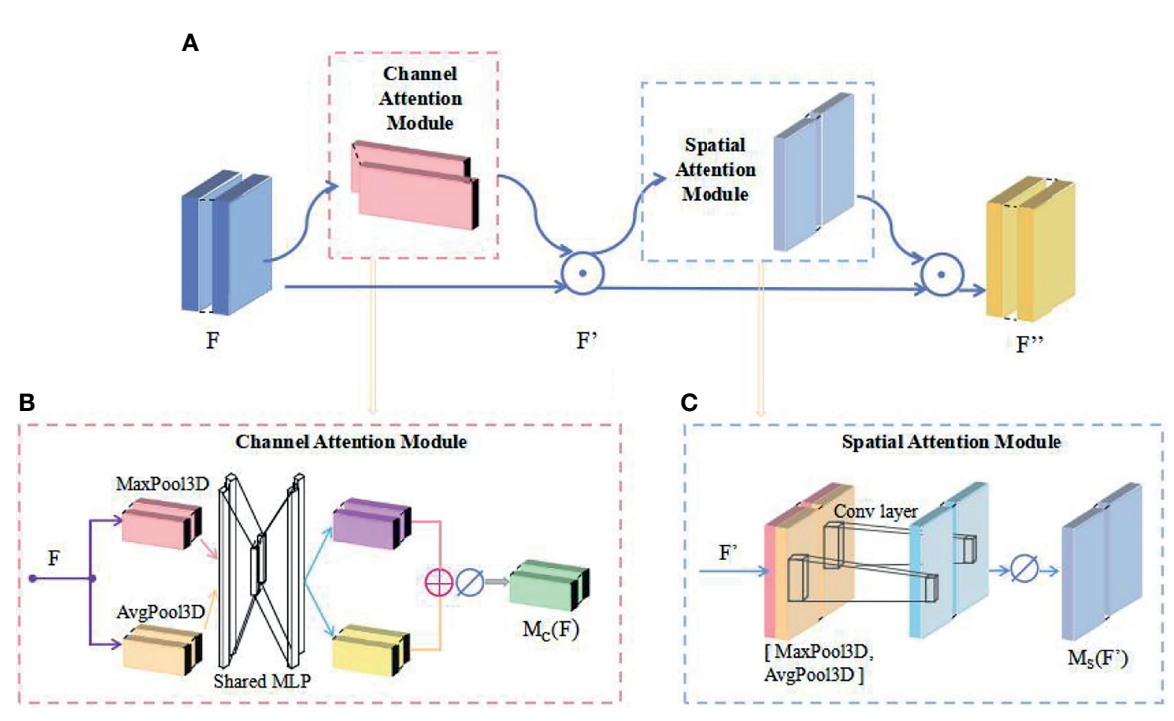

FIGURE 2 | Structure of a 3D CBAM block. (A) The overview of 3D CBAM; (B) Structure of the Channel Attention Module; (C) Structure of the Spatial Attention Module. 
$F^{\prime}$ is used as the input feature map for the spatial attention model as shown in Figure 2C, and the channels are compressed by $3 \mathrm{D}$ global max pooling and $3 \mathrm{D}$ global average pooling in the channel dimension. The two extracted feature maps $F_{\text {avg }}^{S}$ and $F_{\max }^{S}$ are subjected to a channel-based merging operation to obtain a 2-channel feature map, which is subsequently downscaled into a single channel (the shallow blue module in Figure 2 ) by a $7 \times 7$ convolution operation before application of the sigmoid function to generate the output weight $M_{S}\left(F^{\prime}\right)$ in the blue part of the spatial attention module.

$$
\begin{aligned}
M_{S}\left(F^{\prime}\right) & =\sigma\left(f^{7 \times 7}\left(\left[\operatorname{AvgPool} 3 D\left(F^{\prime}\right) ; \operatorname{MaxPool} 3 D\left(F^{\prime}\right)\right]\right)\right) \\
& =\sigma\left(f^{7 \times 7}\left(\left[F_{\text {avg }}^{S} ; F_{\max }^{S}\right]\right)\right)
\end{aligned}
$$

Finally, $M_{S}\left(F^{\prime}\right)$ is multiplied by $F^{\prime}$ to obtain the final output feature map $F^{\prime \prime}$ of the yellow module, as expressed in Equation (3).

$$
F^{\prime}=M_{S}\left(F^{\prime}\right) \otimes F^{\prime}
$$

where $M_{S}\left(F^{\prime}\right)$ denotes the output weight after spatial attention and $\otimes$ denotes element-by-element multiplication.

\section{RA Block}

We propose a residual block with a 3D CBAM, namely, an RA block that is composed of two $3 \times 3 \times 3$ convolutional layers for the pink module, two normalization layers for the purple module, two activation layers for the yellow module, and an attention layer for the green module, as illustrated in Figure 3. Batch normalization $(\mathrm{BN})$ is sensitive to the batch size because the mean and variance are calculated on a single batch. The instance normalization (IN) operation is performed within a single sample and does not depend on the batch. The leaky rectified linear unit (LR) is a variant of ReLU with a variation in response to input fractions of less than 0 , thereby mitigating the sparsity of ReLU and alleviating some of the problems of neuronal death that are caused by ReLU. Therefore, we substitute the instance normalization (IN) and leaky rectified linear unit (LR) functions for the popular batch normalization (BN) and rectified linear unit (ReLU) functions, respectively. The RA block effectively improves the extraction of image feature information by adding a
CBAM after the final convolution layer of the residual block, thereby improving the segmentation accuracy of the network.

\section{DA Block}

Our proposed DA block is shown as a deep blue block in Figure 1, which is composed of multiple green blocks of parallel $3 \times 3 \times 3$ dilated convolution layers with various expansion rates and the orange CBAM blocks, and the multiple parallel feature maps are finally summed to obtain the output feature map of the purple block, as illustrated in Figure 4. Multiscale features are important for the segmentation of brain tumors. Therefore, we propose the DA block for efficiently obtaining feature maps of various sizes through levels of dilated convolutional layers in the spatial pyramid model.

\section{Dice Loss Function}

The BraTS dataset is extremely unbalanced, and convolutional neural networks are very sensitive to unbalanced datasets. Therefore, we use the dice loss function to solve the problem. The dice function is expressed as follows:

$$
L=1-\frac{2 * \sum_{n=1}^{N} p_{\text {pred }} * p_{\text {true }}}{\sum_{n=1}^{N} p_{\text {pred }}^{2}+p_{\text {true }}^{2}+\epsilon}
$$

where $\mathrm{P}_{\text {pred }}$ is the decoder output, $\mathrm{P}_{\text {ture }}$ is the segmentation mask. where summation is voxel-wise, and $€$ is a small constant to avoid zero division.The process of RDAU-Net implementation is as follows. The $3 \mathrm{D}$ brain tumor data of four modalities are convolved twice by two $3 \times 3 \times 3$ layers to increase the number of features in the initial filters. Then, the extracted feature maps are input into the RA block for feature extraction. The CBAM is used to exploit useful information of the input features. The feature maps that are extracted by the first RA block are downsampled by a convolutional layer with a step size of 2 . After four rounds of convolution and downsampling, the extracted feature maps are input into the DA block for feature extraction with various feature sizes. The DA block combines multiple dilated convolutional layers in parallel and incorporates a CBAM dual attention mechanism

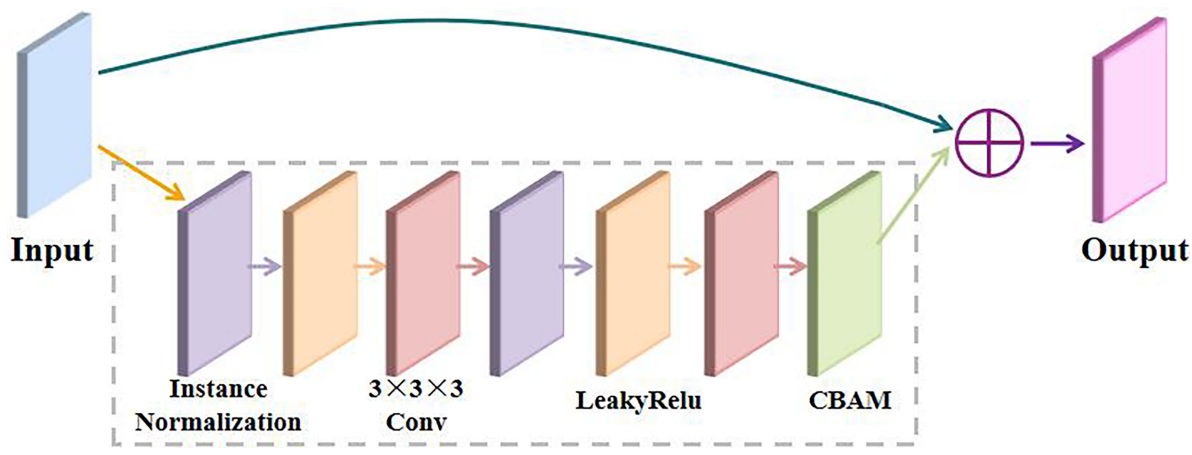

FIGURE 3 | Structure of an RA block. 


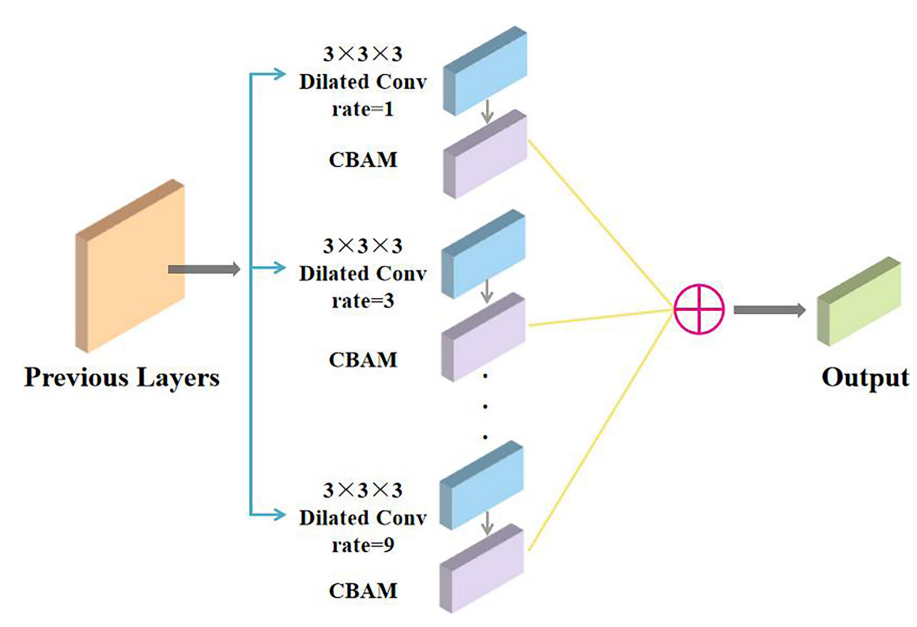

FIGURE 4 | Structure of a DA block.

behind each convolutional layer. This attention pyramid pooling module effectively obtains feature maps of various sizes through levels of expanded convolutional layers in the spatial pyramid model while extracting useful information on channels and spaces to increase the tumor segmentation accuracy. Then, the feature maps that are extracted from the DA block are upsampled by deconvolution. The upsampled feature maps are connected by a skip connection with the feature maps that have undergone feature weighting by the CBAM block in the corresponding layer in the encoder. Our proposed method inserts a CBAM block after each skip connection layer to improve the extraction of channel information and spatial information through tandem channel attention and spatial attention as a way to reduce the redundant information of low-level features. Attention is produced when the skip connection connects the feature maps that are extracted by the encoder directly to the corresponding layers of the decoder. After upsampling, the final prediction result of the network is output through the sigmoid function. Finally, an image of the same size as the input image is generated.

\section{EXPERIMENTS}

\section{Evaluation Indicators}

We use the Dice coefficient, Hausdorff distance, sensitivity, and specificity evaluation metrics to evaluate our experimental results.

The Dice coefficient (Dice) is defined as:

$$
D S C=\frac{2 \times T P}{F P+F N+2 \times T P}
$$

Sensitivity and specificity are defined as:

$$
\begin{aligned}
& \text { Sensitivity }=\frac{T P}{T P+F N} \\
& \text { Specificity }=\frac{T P}{T P+F P}
\end{aligned}
$$

where TP, FP, and FN indicate the true positive, false positive, and false negative values, respectively.

The Hausdorff distance indicates the maximum mismatch between the predicted edge of the tumor segmentation result and the ground-truth boundary.

$$
H D(T, S)=\max \left\{\sup _{x \in X} \inf _{y \in Y} d(x, y), \sup _{y \in Y} \inf _{x \in X} d(x, y)\right\}
$$

where sup and inf denote the upper and lower boundaries, respectively, of the brain tumor region; $\mathrm{x}$ and $\mathrm{y}$ are points on the tumor surface, where $x \in T$ and $y \in P$; and $\mathrm{d}()$ is the distance function.

\section{Experimental Details}

The experiments are carried out on a workstation that is configured with an Intel ${ }^{\circledR}$ Xeon(R) CPU E5-2620 v4 @ $2.10 \mathrm{GHz} \times 32$ and equipped with two 12 GB TITAN Xp graphics cards. The proposed network is tested under the environment of TensorFlow$\mathrm{gpu}==1 \cdot 10.0^{1}$ and Keras $==2 \cdot 2.0^{2}$. For training, the input preprocessed image has an image block size of $4 \times 128 \times 128 \times$ 128. Since the computational complexity of the $3 \mathrm{D}$ image block is too high, we set the batch size to 2 . The experiments are performed using the Adam optimization method, and the initial learning rate is set to $\operatorname{Lr}_{\text {init }}=1 \cdot 10^{-4}$. The learning rate is reduced by $50 \%$ after 15 epochs if the validation loss is not improving, and we regularize with an 12 weight decay of $10^{-5}$. The size of the output prediction segmentation result is also $128 \times 128 \times 128$. Finally, we reshape the data to a size of $240 \times 240 \times 155$ by using the Nilearn package ${ }^{3}$.

\section{Dataset}

Our proposed method is validated on the BraTS 2018 and 2019 (3336) datasets. The BraTS 2018 dataset contains 285 glioma cases, which correspond to $210 \mathrm{HGG}$ patients and 75 LGG patients. The

\footnotetext{
${ }^{1}$ https://github.com/TensorFlow/TensorFlow

${ }^{2}$ https://github.com/keras-team/keras

${ }^{3}$ https://github.com/nilearn/nilearn
} 
validation set contains 66 MRs of patients with unknown tumor grade without real labels. The BraTS 2019 dataset contains 335 glioma cases, which correspond to 259 HGG patients and 76 LGG patients. The validation set contains $125 \mathrm{MRs}$ of patients with unknown tumor grade without real labels. The dataset contains data offour modalities, namely, T1, T2, TIC, and FLAIR, and each MRI is a 3 D image of size $240 \times 240 \times 155$. The task of the segmentation challenge was to segment three tumor subregions: 1) the whole tumor (WT), 2) the tumor core (TC), and 3) the enhancing tumor (ET). Figure 5 shows the modalities for a case in the BraTS 2018 training dataset and the ground truth.

\section{Data Preprocessing}

MRI scans will often show intensity heterogeneity due to variations in the magnetic field. The variations in these mappings are called bias fields, and bias fields can cause problems for classifiers. We used N4ITK for bias field correction of the images, and N4ITK (36) is a modification of the N3 bias field correction method (37). Moreover, the images differ in terms of contrast among these four modes. Therefore, we normalize using the z-score method, namely, we subtract the intensity of each pixel from the average intensity of all pixels in each multimodal image and divide by the standard deviation, while GT is not normalized. Finally, we change the size from $240 \times 240 \times 155$ to $128 \times 128 \times 128$ to reduce the number of parameters of the network.

\section{Ablation Study}

To determine whether the CBAM dual attention module is effective in enhancing the segmentation performance of the network, we perform ablation experiments.

These experiments are conducted with and without the use of the attention module. The scores for the four evaluation metrics on the datasets of the BraTS 2018 and 2019 challenges are obtained separately. As presented in Table 1, the Dice coefficient and Hausdorff distance of the network in which the dual attention mechanism is utilized improved across the board on both the BraTS 2018 and 2019 challenge datasets, especially the Dice score of WT, which improved by $1 \%$ on both datasets, and the Hausdorff distance of ET, which decreased by $0.2 \mathrm{~mm}$ and $0.11 \mathrm{~mm}$, respectively. In addition, the sensitivity of the model in which the attention mechanism is utilized improved by $0.5 \%$ and $0.2 \%$, respectively, on ET. In general, adding CBAMs to the network can effectively improve the performance of the network.

Histograms of various evaluation metrics on the datasets of the BraTS 2018 and 2019 challenges are presented in Figure 6. According to these results, inclusion of the CBAM in the network can effectively improve the segmentation accuracy of the model.

Figure 7 shows the visual segmentation results of our proposed approach on the BraTS 2018 challenge data training set. From Figure 7, we find that our network model can segment various regions of the tumor, especially the ET parts of the tumor, but there are segmentation errors in small places compared with the ground truth. In summary, by comparing our segmentation results with those of RDU-Net (without attention) and the ground truth, we find that our proposed model obtains satisfactory segmentation results with the RA block and the DA block.

\section{Results on the BraTS Challenge Training Data}

We compare the segmentation performance of our approach with those of other typical deep network methods, as shown in Figure 8, on the BraTS 2018 challenge training dataset. The original data in Figure 8 from top to bottom are T1-c; the segmentation results of

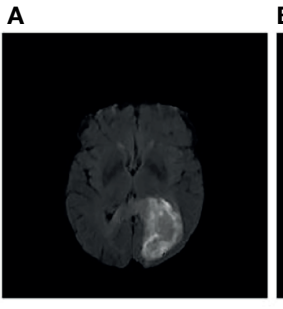

FLAIR

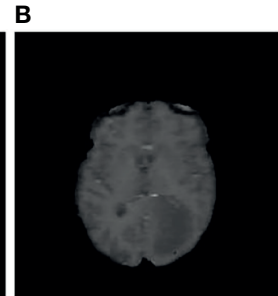

T1

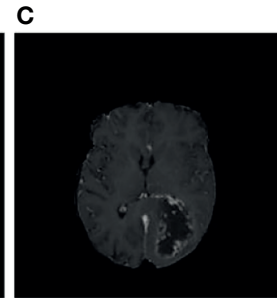

T1C

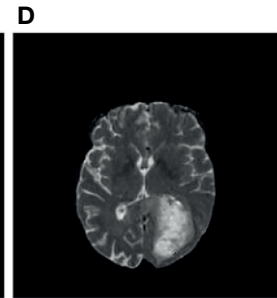

T2

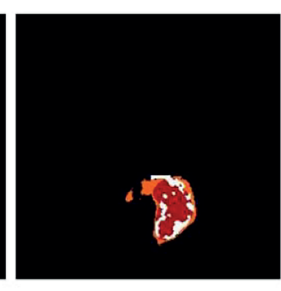

Groundtruth

FIGURE 5 | Schematic diagram of the BRATS 2018 training dataset. From left to right are (A) FLAIR, (B) T1, (C) T1-C, and (D) ground truth, where the colors of the ground truth represent categories of tumor segmentation: orange represents edematous regions, red represents enhancing tumors, and white represents necrotic and nonenhancing tumors.

TABLE 1 | Segmentation results of our proposed network with and without attention mechanism on BraTS 2019 validation set using Dice, Hausdorff distance, specificity, and sensitivity metrics.

\begin{tabular}{|c|c|c|c|c|c|c|c|c|c|c|c|c|}
\hline \multirow[t]{2}{*}{ Models } & \multicolumn{3}{|c|}{ Hausdorff (mm) $\downarrow$} & \multicolumn{3}{|c|}{ Dice $\uparrow$} & \multicolumn{3}{|c|}{ Specificity $\uparrow$} & \multicolumn{3}{|c|}{ Sensitivity $\uparrow$} \\
\hline & WT & TC & ET & WT & TC & ET & WT & TC & ET & WT & TC & ET \\
\hline $\begin{array}{l}\text { RDU-Net } \\
\text { (without CBAM) }\end{array}$ & 6.9705 & 6.1778 & 3.0002 & 0.8895 & 0.9018 & 0.8222 & 0.9894 & 0.9969 & 0.9958 & 0.9169 & 0.9031 & 0.8714 \\
\hline RDAU-Net & 6.8692 & 6.0015 & 2.9980 & 0.9001 & 0.9061 & 0.8311 & 0.9892 & 0.9975 & 0.9966 & 0.9172 & 0.9028 & 0.8730 \\
\hline
\end{tabular}

Results are specified for WT, whole tumor; ET, enhancing tumor; and TC, tumor core. 


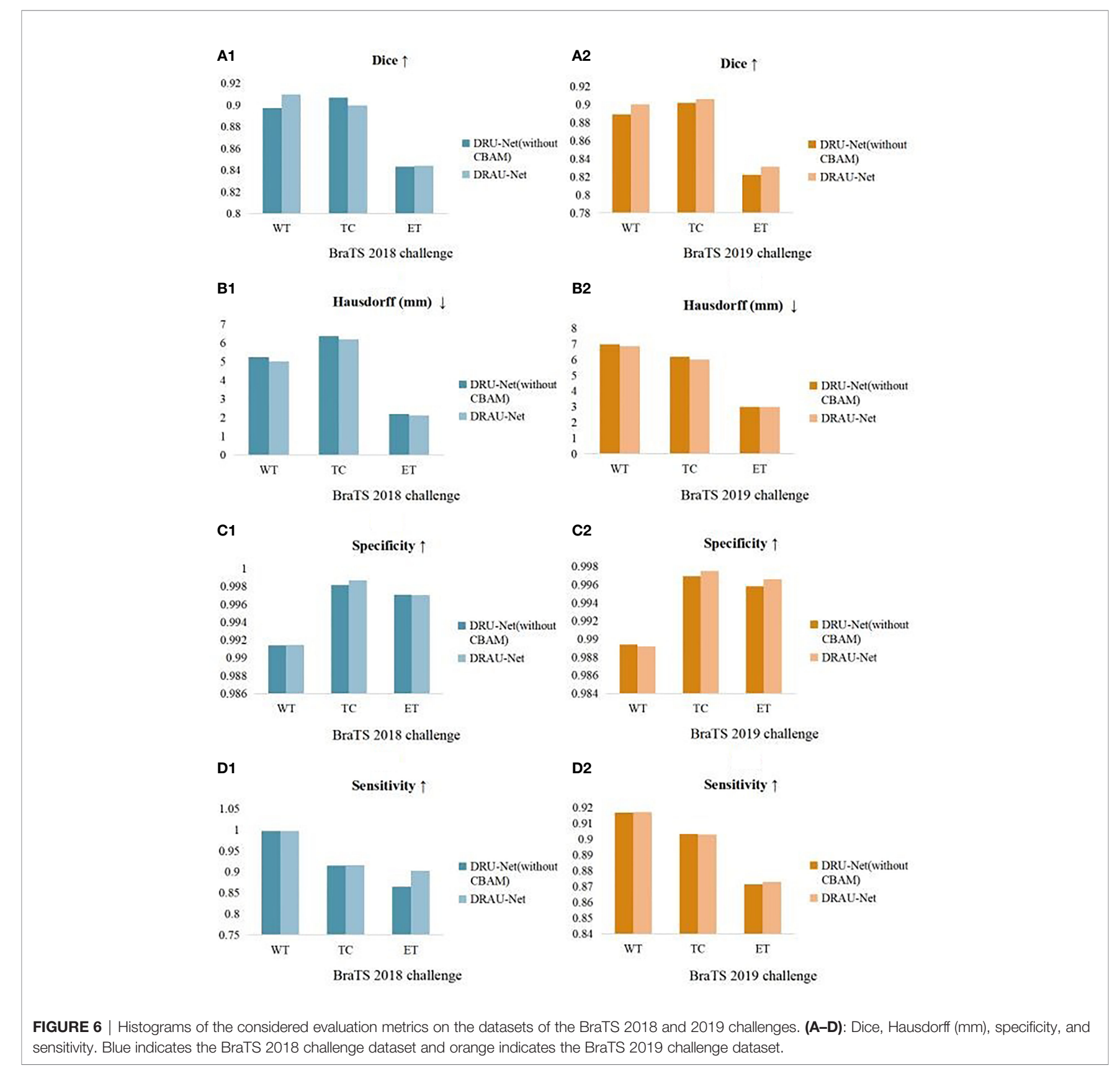

the S3DU-Net (38) model, the AGResU-Net (39) model, and our RDAU-Net method; and the ground truths. By comparing these methods in Figure 8, we observe that the segmentation results of our proposed method, namely, RDAU-Net, are the closest to the ground truth on WT, TC, and ET and that the segmentation results are significantly better than those of the remaining two methods.

Comparison With State-of-the-Art Methods Performance Comparison on the BraTS 2018

\section{Challenge Dataset}

The first-place winner of the Multimodal Brain Tumor Segmentation Challenge (BraTS) 2018 was Myronenko et al., who trained their model using large image blocks with a size of $160 \times 192 \times 128$. As presented in Table 2, the Myronenko et al. (40) method has a higher overall Dice score than other mainstream methods, but our method outperforms Myronenko's method in terms of Dice score by $0.26 \%, 4.7 \%$, and $3 \%$ for WT, TC, and ET, respectively. Our method also outperforms Myronenko's method in terms of Hausdorff distance, which is reduced by $0.7 \mathrm{~mm}$ and $1.7 \mathrm{~mm}$ for TC and ET, respectively. The No New-Net (41) method won second place with only a few minor changes to UNet. As presented in Table 2, our method outperforms their method overall in segmentation. The Dice scores on TC and ET are $5 \%$ and $3.5 \%$ higher than theirs, respectively; the Hausdorff 


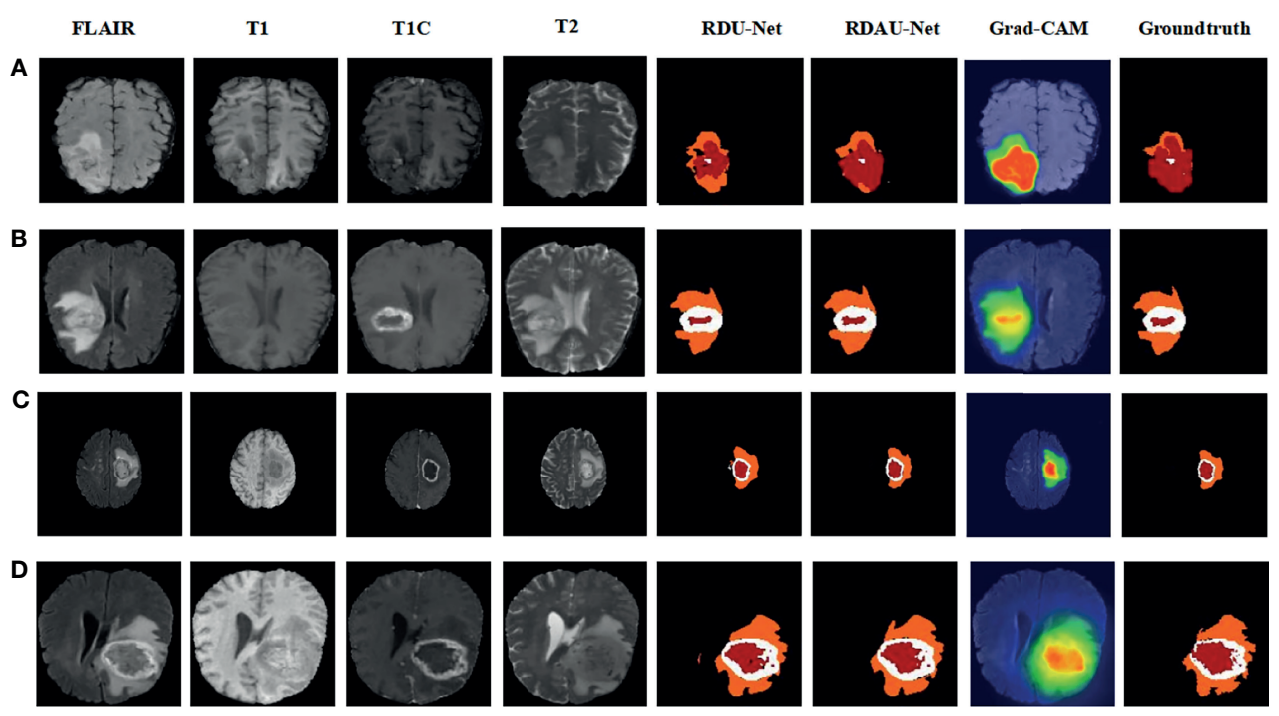

FIGURE 7 | Segmentation results of the proposed method and RDU-Net (without attention) on the BRATS 2018 datasets, the Grad-CAM result and the ground truths. Rows (A-D): Brats18_TCIA01_387_1_93, Brats18_TCIA01_231_1_83, Brats18_CBICA_APR_1_105, Brats18_CBICA_AUN_1_81, where 105, 101, 78, and 73 represent the 105th, 101st, 78th, 73rd slices, respectively, of the MRI data. The colors represent the following categories: $\square$ edema, $\square$ necrosis, and $\square$ enhancing core.

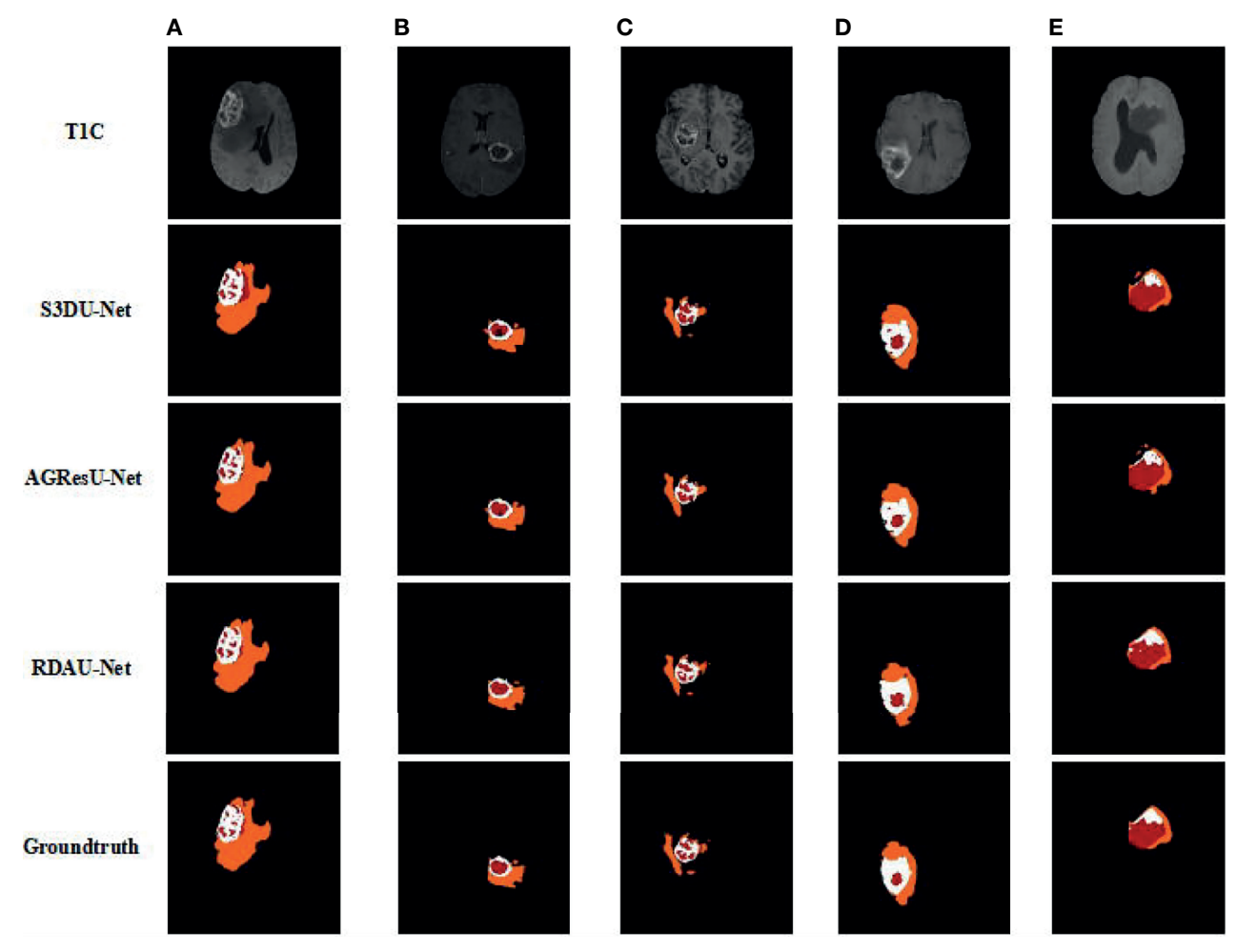

FIGURE 8 | Results of brain tumor segmentation in MRI images using the proposed approach and the ground truths. Columns (A-E) correspond to cases in the Brats 2018 challenge dataset: Brats18_2013_12_1_89, Brats18_CBICA_AQV_1_87, Brats18_TCIA02_117_1_69, Brats18_TCIA04_361_1_90, and Brats18_TCIA05_444_1_84, where $89,87,69,90$, and 84 represent the 89th, 87th, 69th, 90th, and 84 th slices, respectively, of the MRI data. The colors represent the following categories: $\square$ edema, necrosis, and $\square$ enhancing core. 
TABLE 2 | Comparison of performance between our approach and the state-of-the-art methods on BraTS 2018 validation set using Dice, Hausdorff distance, specificity, and sensitivity metrics.

\begin{tabular}{|c|c|c|c|c|c|c|c|c|c|c|c|c|}
\hline Models & \multicolumn{3}{|c|}{ Hausdorff $(\mathrm{mm}) \downarrow$} & \multicolumn{3}{|c|}{ Dice $\uparrow$} & \multicolumn{3}{|c|}{ Specificity $\uparrow$} & \multicolumn{3}{|c|}{ Sensitivity $\uparrow$} \\
\hline Myronenko (40) & 4.41 & 6.84 & 3.82 & 0.9068 & 0.8602 & 0.8173 & - & - & - & - & - & - \\
\hline C-A-Net (42) & 4.1724 & 6.5445 & 2.7162 & 0.9095 & 0.8651 & 0.8136 & 0.9951 & 0.9968 & 0.9983 & 0.9142 & 0.8683 & 0.8135 \\
\hline AGResU-Net (39) & 5.62 & 8.36 & 3.57 & 0.872 & 0.808 & 0.772 & - & - & - & - & - & - \\
\hline S3DU-Net (38) & 4.7165 & 7.7478 & 4.4321 & 0.8935 & 0.8309 & 0.7493 & 0.9927 & 0.9981 & 0.9976 & 0.9290 & 0.8161 & 0.7849 \\
\hline
\end{tabular}

Results are specified for WT, whole tumor; ET, enhancing tumor; and TC, tumor core. The results are marked in bold.

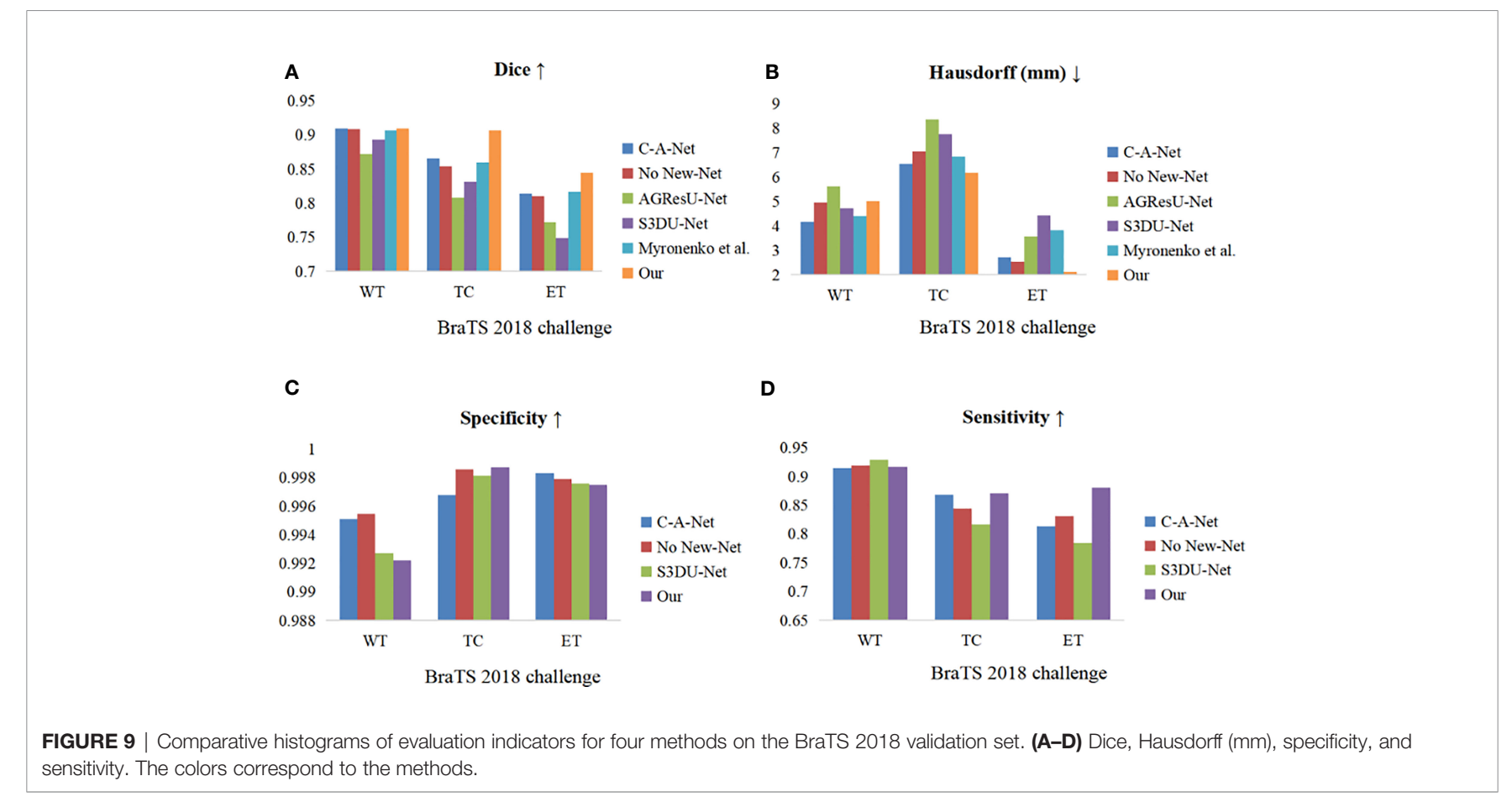

TABLE 3 | Comparison of performance between our approach and the state-of-the-art methods on BraTS 2019 validation set using Dice, Hausdorff distance, specificity, and sensitivity metrics.

\begin{tabular}{|c|c|c|c|c|c|c|c|c|c|c|c|c|}
\hline Models & \multicolumn{3}{|c|}{ Hausdorff $(\mathrm{mm}) \downarrow$} & \multicolumn{3}{|c|}{ Dice $\uparrow$} & \multicolumn{3}{|c|}{ Specificity $\uparrow$} & \multicolumn{3}{|c|}{ Sensitivity $\uparrow$} \\
\hline Two-stage cascaded U-Net (43) & 4.4438 & 5.8620 & 3.2055 & 0.9082 & 0.8632 & 0.8020 & 0.9943 & 0.9974 & 0.9984 & 0.9237 & 0.8622 & 0.8038 \\
\hline Hamhanghala et al. (44) & 6.9 & 8.4 & 4.6 & 0.8965 & 0.7901 & 0.7665 & 0.9939 & 0.9976 & 0.9985 & 0.9132 & 0.7771 & 0.7688 \\
\hline DDU-Nets46] & 4.874 & 8.013 & 3.376 & 0.898 & 0.793 & 0.780 & 0.994 & 0.996 & 0.998 & 0.903 & 0.808 & 0.791 \\
\hline Myronenko et al. (46) & 5.89 & 6.562 & 3.921 & 0.8900 & 0.8340 & 0.8000 & - & - & - & - & - & - \\
\hline 3D U-Net (47) & 7.357 & 5.667 & 5.994 & 0.807 & 0.894 & 0.737 & 0.996 & 0.995 & 0.998 & 0.826 & 0.897 & 0.766 \\
\hline
\end{tabular}

Results are specified for WT, whole tumor; ET, enhancing tumor; and TC, tumor core. The results are marked in bold.

distances on TC and ET are $0.9 \mathrm{~mm}$ and $0.4 \mathrm{~mm}$ shorter than theirs, respectively; and the sensitivity scores on TC and ET are $3 \%$ and $5 \%$ better than theirs, respectively. C-A-Net (42) is a single-channel multitask network that combines multiple $\mathrm{CNN}$ structures. According to Table 2, our network outperforms the
C-A-Net method on all metrics except the Hausdorff distance score on WT, and the Dice scores on TC, and ET are improved by $5 \%$, and $3.5 \%$, respectively. Our sensitivity score on ET is nearly $5 \%$ higher than that of the C-A-Net method. The AGResU-Net method (39) integrates the residual module and 

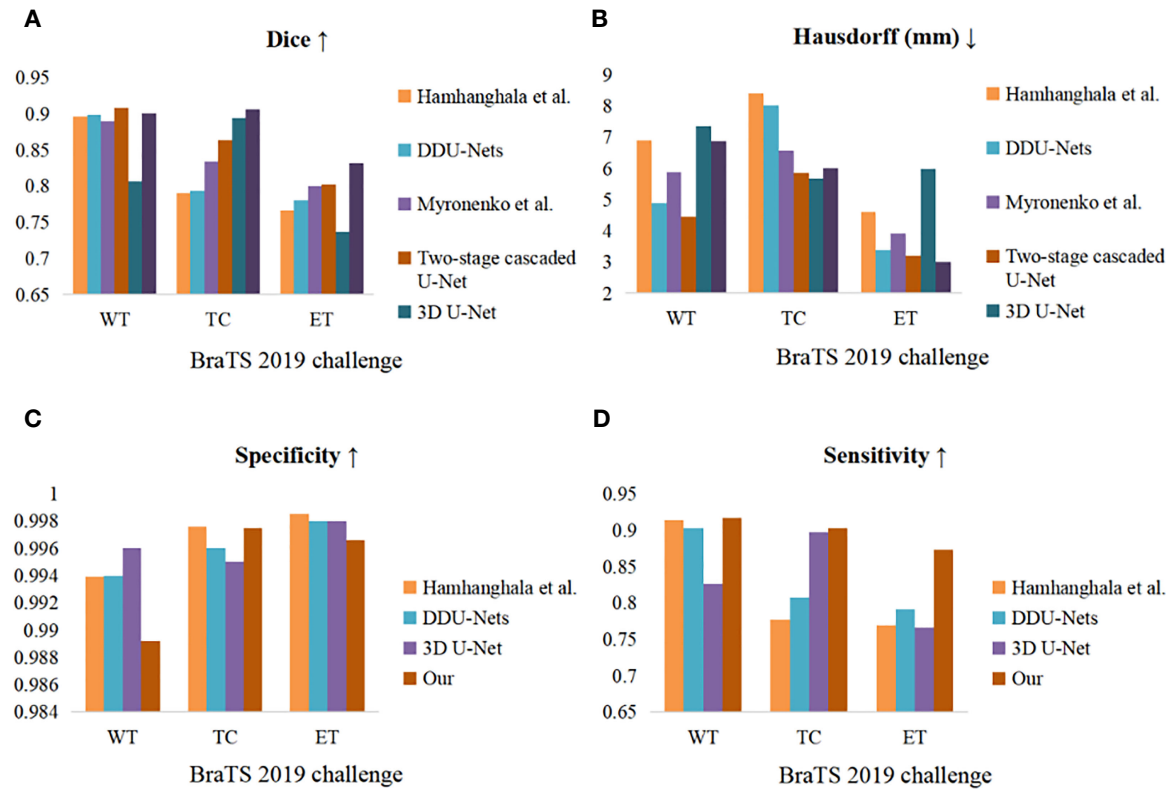

FIGURE 10 | Comparative histograms of evaluation indicators for four methods on the BraTS 2019 validation set. (A-D) Dice, Hausdorff (mm), specificity, and sensitivity. The colors correspond to the methods.

attention gates in the original U-Net. As presented in Table 2, our method outperforms the network on all metrics, especially the Hausdorff distance on TC: our method obtains a Hausdorff distance that is $2.1 \mathrm{~mm}$ shorter than that obtained by the AGResU-Net method, and our method obtains Dice scores that are improved by $9 \%$ and $7 \%$ on TC and ET, respectively. The S3DU-Net method (38) is a module in which the convolutional block in U-Net is changed to a $3 \mathrm{D}$ convolution with three parallel branches. It scores well on the sensitivity metric on WT, but our network outperforms that network overall. Compared with the S3DU-Net method, our method improves the Dice score by $7 \%$ and $9 \%$ on TC and ET, respectively; reduces the Hausdorff distance by $1.5 \mathrm{~mm}$ and $2.3 \mathrm{~mm}$ on TC and ET, respectively; and improves the sensitivity by $5 \%$ and $9 \%$ on TC and ET, respectively. The histograms in Figure 9 compare several methods in terms of various metrics. According to the comparison in Figure 9, our method is highly competitive.

\section{Performance Comparison on the BraTS 2019 Challenge Dataset}

For the BraTS 2019 challenge dataset, RDAU-Net still achieved the best results in terms of the Hausdorff distance metric in the tumor core region and the Dice coefficients of TC and ET and performed very competitively in terms of other metrics. The twostage cascaded U-Net (43) won first place in the BraTS 2019 challenge. Compared with the champion team, the Dice scores and the sensitivity scores on ET and TC of our method are almost $3 \%$ and $4.3 \%$ higher and $7 \%$ and $4 \%$ higher, respectively. The Hausdorff distance of the ET subregion that was obtained using our method is approximately $0.2 \mathrm{~mm}$ shorter.
Hamhanghala et al. (44) used generative adversarial networks to expand the data. Although the training sample was expanded with synthetic data, this approach did not yield more prominent results. DDU-Nets (45) contain three models of distributed dense connectivity. DDU-Nets are not particularly effective overall, although they slightly outperform our method in terms of Hausdorff distance scores on WT and ET. Myronenko et al. (46) further improved the loss function based on 2018 by introducing the focal loss and using eight $32 \mathrm{G}$ video cards for training. As presented in Table 3, our network still outperforms Myronenko et al.'s method on all other metrics, except for a slightly lower Hausdorff distance score on WT than their method. The 3D U-Net (47) is designed mainly to handle block diagrams. From Table 3, our method outperforms the 3D U-Net method on all metrics except for the Dice score on TC and the specificity scores on WT and ET, which are slightly lower than those of the 3D U-Net method. The histograms in Figure 10 clearly compare the considered methods in terms of various metrics. According to the comparison, our method is still very competitive, even on the newer dataset.

\section{CONCLUSIONS}

We propose a new method, namely, RDAU-Net that is based on an improved ResU-Net for brain tumor segmentation in MRI. We add DA blocks to expand the receptive field and obtain image information of various sizes and insert a CBAM block after each skip connection layer to improve the extraction of channel information and spatial information to reduce the 
redundant information of low-level features. By conducting experiments on the BraTS 2018 and BraTS 2019 datasets, we find that the use of an RA block instead of a convolutional layer, the inclusion of a DA block in the network, and the insertion of 3D CBAM blocks can effectively improve the performance of the network. RDAU-Net has more obvious advantages than the SOTA method. However, the performance of the method in the WT region still has substantial room for improvement, and we hope to solve this problem through postprocessing of the network. In conclusion, the method has greater advantages in segmenting subregions of brain tumors and can be effectively applied to clinical research.

\section{DATA AVAILABILITY STATEMENT}

Publicly available datasets were analyzed in this study. This data can be found here: https://doi.org/10.7937/K9/TCIA.2017.KLXWJJ1Q.

\section{AUTHOR CONTRIBUTIONS}

JW provided guidance on the content of the article as well as the writing of the article. ZY writes articles and conducts experiments. ZL and JR completed the work of collecting references. $\mathrm{YZ}$ provided guidance on medical knowledge and medical image knowledge. GY provided guidance on the experiments done in the article. All authors are approved for publication.

\section{FUNDING}

This work was jointly supported by the Shandong University Science and technology project (No.J17KA0 82, No. J16LN21), the National Natural Science Foundation of China (No.61401259), the China Postdoctoral Science Foundation (No.2015M582128).

\section{REFERENCES}

1. Siegel R, Naishadham D, Jemal A. Cancer Statistics, 2013. CA Cancer J Clin (2013) 63(1):11-30. doi: 10.3322/caac.21166

2. Yang D. Standardized MRI Assessment of High-Grade Glioma Response: A Review of the Essential Elements and Pitfalls of the RANO Criteria. NeuroOncology Pract (2014) 3(1):59-67. doi: 10.1093/nop/npv023

3. Reznek RH. CT/MRI of Neuroendocrine Tumours. Cancer Imaging (2006) 6:163-77. doi: 10.1102/1470-7330.2006.9037

4. Bauer S, Wiest R, Nolte L, Reyes M. A Survey of MRI-Based Medical Image Analysis for Brain Tumor Studies. Phys Med Biol (2013) 58(13):97-129. doi: 10.1088/0031-9155/58/13/R97

5. Pereira S, Pinto A, Alves V, Silva C. Brain Tumor Segmentation Using Convolutional Neural Networks in MRI Images. IEEE Trans Med Imaging (2016) 35(5):1240-51. doi: 10.1109/TMI.2016.2538465

6. Nie D, Wang L, Adeli E, Lao C, Lin W, Shen D. 3-D Fully Convolutional Networks for Multimodal Isointense Infant Brain Image Segmentation. IEEE Trans Cybern (2019) 49(3):1123-36. doi: 10.1109/TCYB.2018.2797905

7. Chen S, Ding C, Liu M. Dual-Force Convolutional Neural Networks for Accurate Brain Tumor Segmentation. Pattern Recognit (2019) 88:90-100. doi: 10.1016/j.patcog.2018.11.009

8. Zhuge Y, >Ning H, Mathen P, Cheng J, Krauze A, Camphausen K, et al. Automated Glioma Grading on Conventional MRI Images Using Deep Convolutional Neural Networks. Med Phys (2020) 47(7):3044-53. doi: $10.1002 / \mathrm{mp} .14168$

9. Zhong Z, Fan B, Duan J, Wang L, Ding K, Xiang S, et al. Discriminant Tensor Spectral-Spatial Feature Extraction for Hyperspectral Image Classification. IEEE Geosci Remote Sens Lett (2015) 12(5):1028-32. doi: 10.1109/ LGRS.2014.2375188

10. Simonyan K, Zisserman A. Very Deep Convolutional Networks for LargeScale Image Recognition. In: 3rd Int. Conf. Learn. Represent. ICLR 2015 - Conf. Track Proc. San Diego, CA, USA: arXiv (2015). p. 1-14.

11. Krizhevsky A, Sutskever I, Hinton GE. ImageNet Classification With Deep Convolutional Neural Networks. Commun ACM (2017) 60(6):84-90. doi: $10.1145 / 3065386$

12. Srigurulekha K, Ramachandran V. Food Image Recognition Using CNN. In: 2020 Int. Conf. Comput. Commun. Informatics, ICCCI 2020 (2020). Coimbatore, India: IEEE. doi: 10.1109/ICCCI48352.2020.9104078

13. Qamar S, Jin H, Zheng R, Ahmad P. 3d Hyper-Dense Connected Convolutional Neural Network for Brain Tumor Segmentation. In: Proc. 2018 14th Int. Conf. Semant. Knowl. Grids, SKG 2018. Guangzhou, China: IEEE (2018). p. 123-30. doi: 10.1109/SKG.2018.00024

14. Wang G, Zuluaga M, Li W, Pratt R, Patel P, Aertsen M, et al. DeepIGeoS: A Deep Interactive Geodesic Framework for Medical Image Segmentation. IEEE Trans Pattern Anal Mach Intell (2019) 41(7):1559-72. doi: 10.1109/ TPAMI.2018.2840695

15. Dolz J, Gopinath K, Yuan J, Lombaert H, Desrosiers C, Ben A. HyperDenseNet: A Hyper-Densely Connected CNN for Multi-Modal Image Segmentation. IEEE Trans Med Imaging (2019) 38(5):1116-26. doi: 10.1109/TMI.2018.2878669

16. Wang W, Gang J. Application of Convolutional Neural Network in Natural Language Processing. In: Proc. 2018 Int. Conf. Inf. Syst. Comput. Aided Educ. ICISCAE 2018. Changchun, China: IEEE. (2019). p. 64-70. doi: 10.1109/ ICISCAE.2018.8666928

17. Jin N, Wu J, Ma X, Yan K, Mo Y. Multi-Task Learning Model Based on MultiScale CNN and LSTM for Sentiment Classification. IEEE Access (2020) 8:77060-72. doi: 10.1109/ACCESS.2020.2989428

18. Long J, Shelhamer E, Darrell T, Berkeley U. Fully Convolutional Networks for Semantic Segmentation. In: 2015 IEEE Conference on Computer Vision and Pattern Recognition (CVPR). Boston, America: IEEE (2015). p. 640-51. doi: 10.1109/CVPR.2015.7298965

19. Ronneberger O, Fischer P, Brox T. U-Net: Convolutional Networks for Biomedical Image Segmentation. In: Med. Image Comput. Comput. Interv. , MICCAI. Munich, Germany: arXiv. (2015). p. 234-41. doi: 10.1007/978-3319-24574-4_28

20. Oktay O, Schlemper J, Le Folgoc L, Lee M, Heinrich M, Misawa K, et al. Attention U-Net: Learning Where to Look for the Pancreas. arXiv(Midl) (2018) 1-10.

21. Shankaranarayana SM, Ram K, Mitra K, Sivaprakasam M. Joint Optic Disc and Cup Segmentation Using Fully Convolutional and Adversarial Networks. Lect Notes Comput Sci (2017) 10554 LNCS:168-76. doi: 10.1007/978-3-31967561-9_19

22. Bahdanau D, Cho KH, Bengio Y. Neural Machine Translation by Jointly Learning to Align and Translate. In: 3 rd International Conference on Learning Representations, ICLR 2015 - Conference Track Proceedings. San Diego, CA, USA: arXiv. (2015). p. 1-15.

23. Vaswani A, Shazeer N, Parmar N, Uszkoreit J, Jones L, Gomez AN, et al. Attention is All You Need. Adv Neural Inf Process Syst (2017) 5999-6009.

24. Galassi A, Lippi M, Torroni P. Attention in Natural Language Processing. IEEE Trans Neural Networks Learn Syst (2020) 32(10):4291-308. doi: 10.1109/ tnnls.2020.3019893

25. Jiang L, Sun X, Mercaldo F, Santone A. DECAB-LSTM: Deep Contextualized Attentional Bidirectional LSTM for Cancer Hallmark Classification. KnowledgeBased Syst (2020) 210:106486-95. doi: 10.1016/j.knosys.2020.106486 
26. de S. Correia A, Colombini EL. Attention, Please! A Survey of Neural Attention Models in Deep Learning. arXiv (2021).

27. Niu Z, Zhong G, Yu H. A Review on the Attention Mechanism of Deep Learning. Neurocomputing (2021) 452:48-62. doi: 10.1016/j.neucom.2021.03.091

28. Shao Y, Lan J, Liang Y, Hu J. Residual Networks With Multi-Attention Mechanism for Hyperspectral Image Classification. Arab J Geosci (2021) 14 (4):252-70. doi: 10.1007/s12517-021-06516-6

29. Hu J, Shen L, Sun G. Squeeze-And-Excitation Networks. In: Proceedings of the IEEE Computer Society Conference on Computer Vision and Pattern Recognition. Salt Lake City, Utah: IEEE (2018). p. 7132-41. doi: 10.1109/CVPR.2018.00745

30. Wang Q, Wu B, Zhu P, LiP, Zuo W, Hu Q. ECA-Net: Efficient Channel Attention for Deep Convolutional Neural Networks. In: Proceedings of the IEEE Computer Society Conference on Computer Vision and Pattern Recognition. Seattle, Washington: IEEE/ CVF Conference on Computer Vision and Pattern Recognition (CVPR) IEEE (2020). p. 11531-9. doi: 10.1109/CVPR42600.2020.01155

31. Fu J, Liu J, Tian H, Li Y, Bao Y, Fang Z, et al. Dual Attention Network for Scene Segmentation. In: Proceedings of the IEEE Computer Society Conference on Computer Vision and Pattern Recognition. Long Beach, CA: IEEE (2019). p. 3141-9. doi: 10.1109/CVPR.2019.00326

32. Woo S, Park J, Lee J, Kweon I. CBAM: Convolutional Block Attention Module. arXiv (2018) 1:3-19. doi: 10.1007/978-3-030-01234-2

33. Bakas S, Akbari H, Sotiras A, Bilello M, Rozycki M, Kirby J, et al. Advancing The Cancer Genome Atlas Glioma MRI Collections With Expert Segmentation Labels and Radiomic Features. Sci Data (2017) 4(March):113. doi: $10.1038 /$ sdata.2017.117

34. Bakas S, Reyes M, Jakab A, Bauer S, Rempfler M, Crimi A, et al. Identifying the Best Machine Learning Algorithms for Brain Tumor Segmentation, Progression Assessment, and Overall Survival Prediction in the BRATS Challenge. arXiv (2018) 1-49.

35. Bakas S, Akbari H, Sotiras A, Bilello M, Rozycki M, Kirby JS, et al. Segmentation Labels and Radiomic Features for the Pre-Operative Scans of the TCGA-GBM Collection. Cancer Imaging Arch (2017).

36. Tustison NJ, Avants BB, Cook PA, Zheng Y, Egan A, Yushkevich PA, et al. N4ITK: Improved N3 Bias Correction. IEEE Trans Med Imaging (2010) 29 (6):1310-20. doi: 10.1109/TMI.2010.2046908

37. Larsen CT, Eugenio Iglesias J, Van Leemput K. N3 Bias Field Correction Explained as a Bayesian Modeling Method. Lect Notes Comput Sci (2014) 8677:1-12. doi: 10.1007/978-3-319-12289-2_1

38. Zhang J, Jiang Z, Dong J, Hou Y, Liu B. Attention Gate ResU-Net for Automatic MRI Brain Tumor Segmentation. IEEE Access (2020) 8:58533-45. doi: 10.1109/ ACCESS.2020.2983075

39. Chen W, Liu B, Peng S, Sun J, Qiao X. S3D-UNet: Separable 3d U-Net for Brain Tumor Segmentation Wei. Granada, Spain: Springer International Publishing (2018). doi: 10.1007/978-3-030-11726-9_32
40. Myronenko A. 3d MRI Brain Tumor Segmentation Using Autoencoder Regularization. Int MICCAI Brainlesion Workshop (2018) 11384:311-20. doi: 10.1007/978-3-030-11726-9

41. Isensee F, Kickingereder P, Wick W, Bendszus M, Maier-Hein K. No NewNet. Lect Notes Comput Sci (2019) 11384:234-44. doi: 10.1007/978-3-03011726-9_21

42. Zhou C, Chen S, Ding C, Tao D. Learning Contextual and Attentive Information for Brain Tumor Segmentation, in Lecture Notes in Computer Science (including subseries Lecture Notes in Artificial Intelligence and Lecture Notes in Bioinformatics). Springer International Publishing (2019) 11384:497507. doi: 10.1007/978-3-030-11726-9

43. Jiang Z, Ding C, Liu M, Tao D. Two-Stage Cascaded U-Net: 1st Place Solution to Brats Challenge 2019 Segmentation Task. Lecture Notes Comput Sci (2020) 11992:231-41. doi: 10.1007/978-3-030-46640-4_22

44. Hamghalam M, Lei B, Wang T. Brain Tumor Synthetic Segmentation in 3d Multimodal MRI Scans. Lect Notes Comput Sci (2020) 11992:153-62. doi: 10.1007/978-3-030-46640-4_15

45. Zhang H, Li J, Shen M, Wang Y, Yang G. DDU-Nets: Distributed Dense Model for 3D MRI Brain Tumor Segmentation. Lect Notes Comput Sci (2020) 11993:208-17. doi: 10.1007/978-3-030-46643-5_20

46. Myronenko A, Hatamizadeh A. Robust Semantic Segmentation of Brain Tumor Regions From 3D MRIs. Lect Notes Comput Sci (2020) 11993:82-9. doi: 10.1007/978-3-030-46643-5_8

47. Wang F, Jiang R, Zheng L, Meng C, Biswai B. 3d U-Net Based Brain Tumor Segmentation and Survival Days Prediction. Lecture Notes Comput Sci (2020) 11992:131-41. doi: 10.1007/978-3-030-46640-4_13

Conflict of Interest: The authors declare that the research was conducted in the absence of any commercial or financial relationships that could be construed as a potential conflict of interest.

Publisher's Note: All claims expressed in this article are solely those of the authors and do not necessarily represent those of their affiliated organizations, or those of the publisher, the editors and the reviewers. Any product that may be evaluated in this article, or claim that may be made by its manufacturer, is not guaranteed or endorsed by the publisher.

Copyright $\odot 2022$ Wang, Yu, Luan, Ren, Zhao and Yu. This is an open-access article distributed under the terms of the Creative Commons Attribution License (CC BY). The use, distribution or reproduction in other forums is permitted, provided the original author(s) and the copyright owner(s) are credited and that the original publication in this journal is cited, in accordance with accepted academic practice. No use, distribution or reproduction is permitted which does not comply with these terms. 